\title{
A 3D QCT Technique of the Thoracic and Lumbar Spine: Integral Volume and Intervertebral Disc Space Increase and BMD decreases from T6 to L4
}

\section{O. Museyko', Johannes Scheitacker', Axel Heinemann², Mattias Krause ${ }^{3}$, Reinhard Barkmann ${ }^{4}$, Michael Amling ${ }^{3}$, Claus Glüer ${ }^{4}$, Klaus Püschel ${ }^{2}$, K. Engelke ${ }^{1}$}

1 Institute of Medical Physics (IMP), University of Erlangen-Nuremberg, Germany

Institute for Forensic Medicine, University of Hamburg, Hamburg, Germany

${ }^{3}$ Institute for Osteology and Biomechanics, University of Hamburg, Hamburg, Germany

${ }^{4}$ Molecular Imaging North Competence Center, University of Kiel, Kiel, Germany

\section{INTRODUCTION}

QCT of the spine is typically restricted to the BMD analysis of the lumbar vertebrae. However, fractures frequently occur in the thoracolumbar region. Also the load distribution in the spine may depend on the intervertebral disc space (IDS), an approximation of the intervertebral disc, which itself cannot be reliably assessed with X-ray based methods (Fig.1). Thus, finite element analysis of vertebral column should be more accurate if the vertebral discs are considered in the modelling process. On the other hand, IDS parameters such as volume may be of interest as additional fracture risk predictors for osteoporotic patients [2].

In this cadaver study, IDS volume and integral and trabecular BMD and volume of the vertebral bodies T6 - L4 have been analyzed.

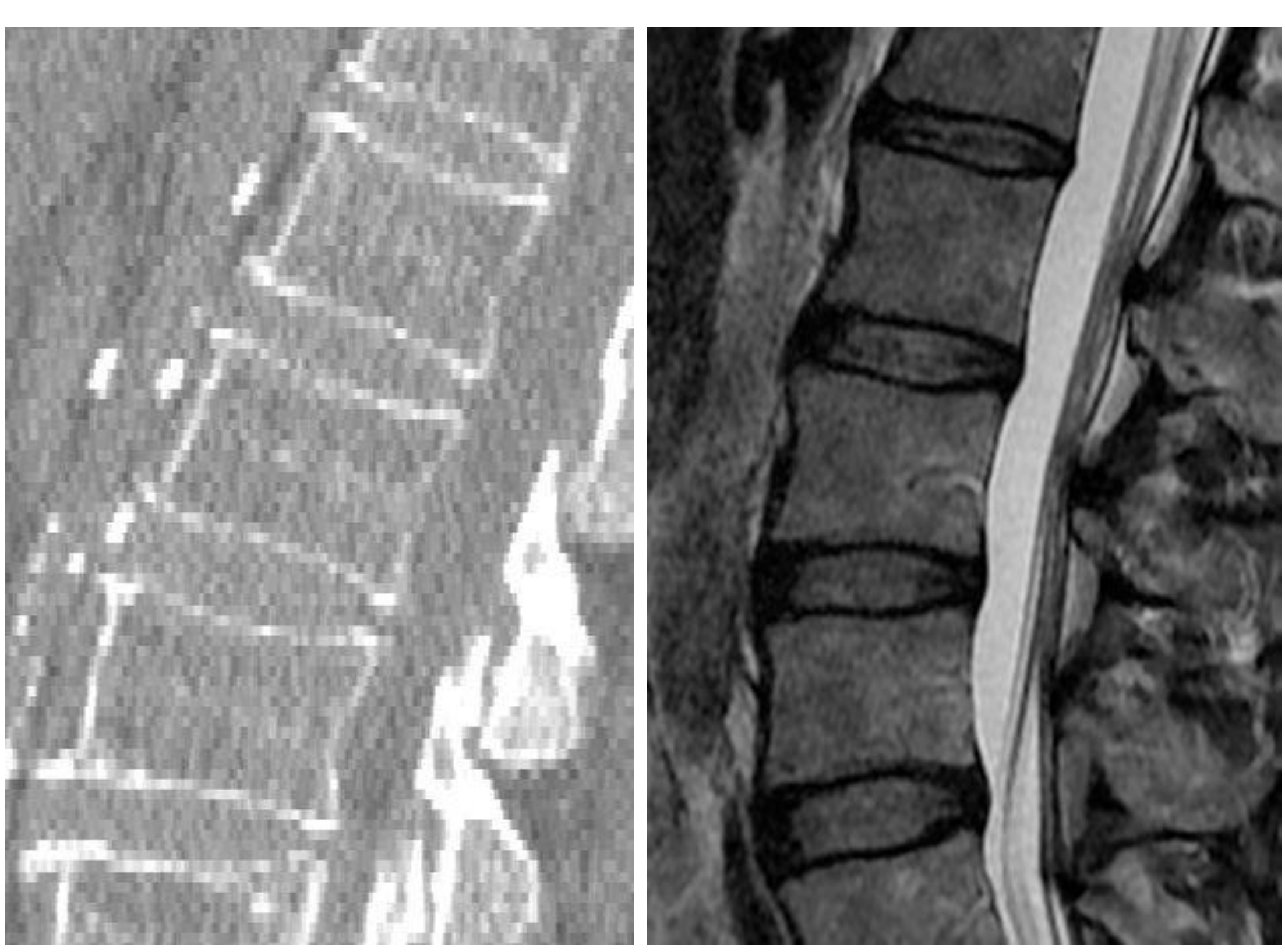
Figure 1: Illustration of the discs in t CT (left) and MRI (right). One can clearly distinguish the borders of individual discs in MR, whereas in $\mathrm{CT}$ the discs are indistinguishable surrounding soft tissue.

\section{MATERIALS AND METHODS}

QCT Datasets 16 human cadavers aged 65-90 were used for the study. All acquisitions were made on a Philips MX8000 scanner with the following parameters: $120 \mathrm{kV}, 100 \mathrm{mAs}$, pitch 1, slice thickness 1.3 $\mathrm{mm}$, field of view $15-16 \mathrm{~cm}$. The scan range included vertebrae from T6 to L4 with adjacent endplates of T5 and L5. Vertebrae with fractures, injected cement, or internal metal hardware were excluded. The IDS was only analyzed if none of the two bordering vertebrae was excluded.

121 vertebrae and 77 IDS were included in the analysis.

Segmentation of lumbar and thoracic vertebrae An automated segmentation technique (with optional operator interaction) implemented in the Medical Image Analysis Framework (MIAF-Spine) [1], was used to obtain boundaries of integral and trabecular compartments of the vertebral bodies. Originally developed for lumbar vertebrae, MIAF-Spine was modified to allow for segmentation of thoracic vertebrae as well, with ribs excluded (Fig.2). Here the spine segment (T6 to L4) was analysed.

Segmentation of IDS IDS was defined as the volume between the endplates of two adjacent non-fractured vertebrae and a lateral surface connecting the ridge points of the endplates. Specifically, two compartments were distinguished: core (Fig. 3 and 4, purple VOI) and extended (Fig.4, green VOI). These approximate nucleus pulposus and anulus fibrosus, respectively, which have different structural and material properties. In the current study, we restricted the analysis to the union of both compartments.

Statistical analysis A correlation between IDS volume and BMD of the vertebra below was performed. Trends in values for IDS volume and vertebral bodies and for BMD of the latter were analyzed depending on the vertebral level with normalization to T12 (if available).

\section{RESULTS}

Difference in IDS volume and in trabecular and integral BMD normalized to the respective values of $\mathrm{T} 12$, are shown in the table With few exceptions, volume increases and trabecular BMD decreases from cranial to caudal vertebrae whereas integral BMD is constant in the thoracic vertebrae and decreases in the lumbar vertebrae monotonically.

The correlation coefficient between IDS volume and integral volume of the vertebra was $r=0.79$ for the thoracic and $r=0.64$ for the lumbar spine $(p<0.01$ for both $r$-values, $r=0.86$ for the pooled data). Also, IDS volume significantly correlated with integral and trabecular BMD of the vertebra, see Figures 5 and 6.

\section{RESULTS (continued)}

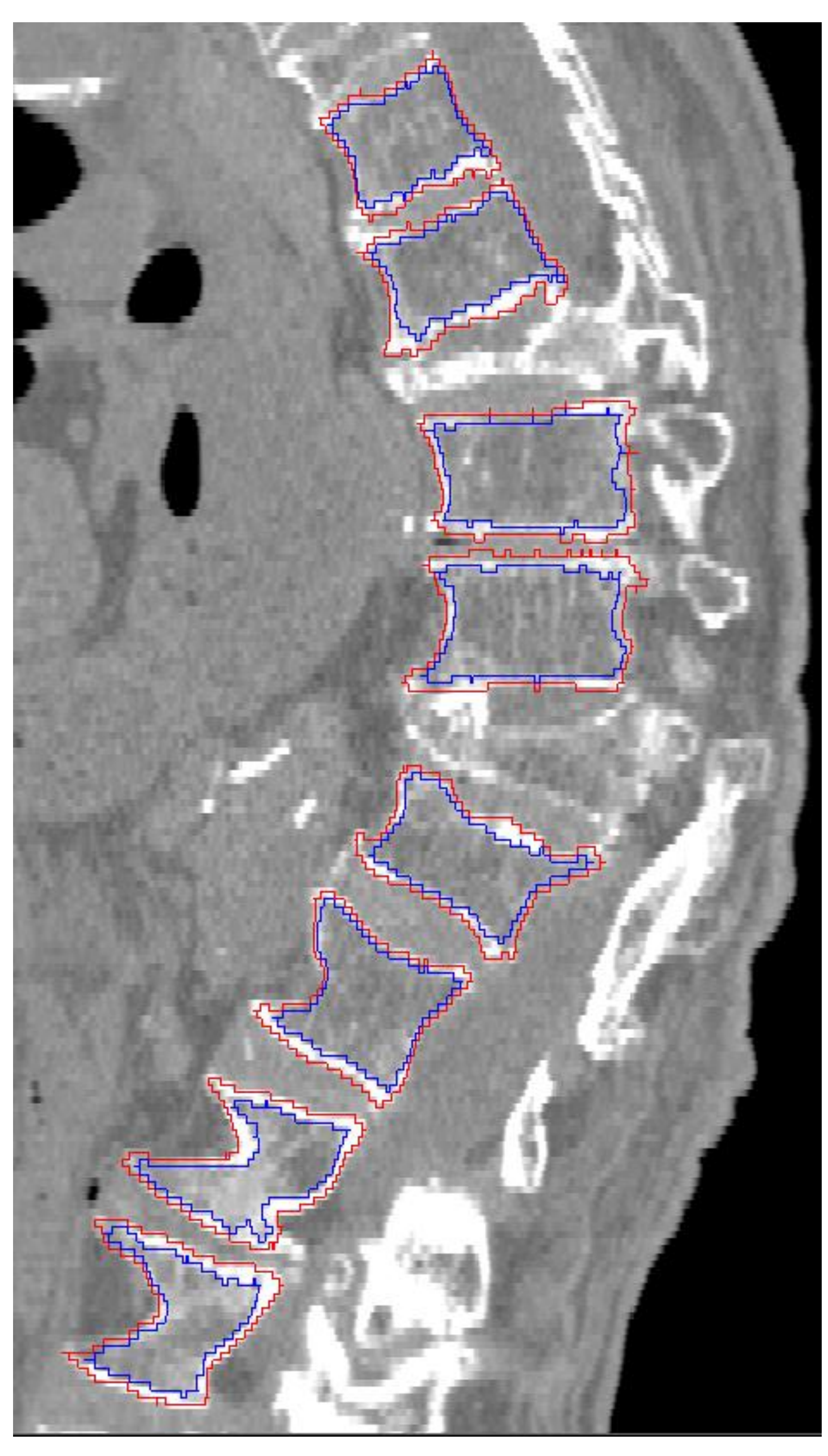

Figure 2: Example of the analyzed spine segment with segmentation of unfractured blue: trabecular compartment

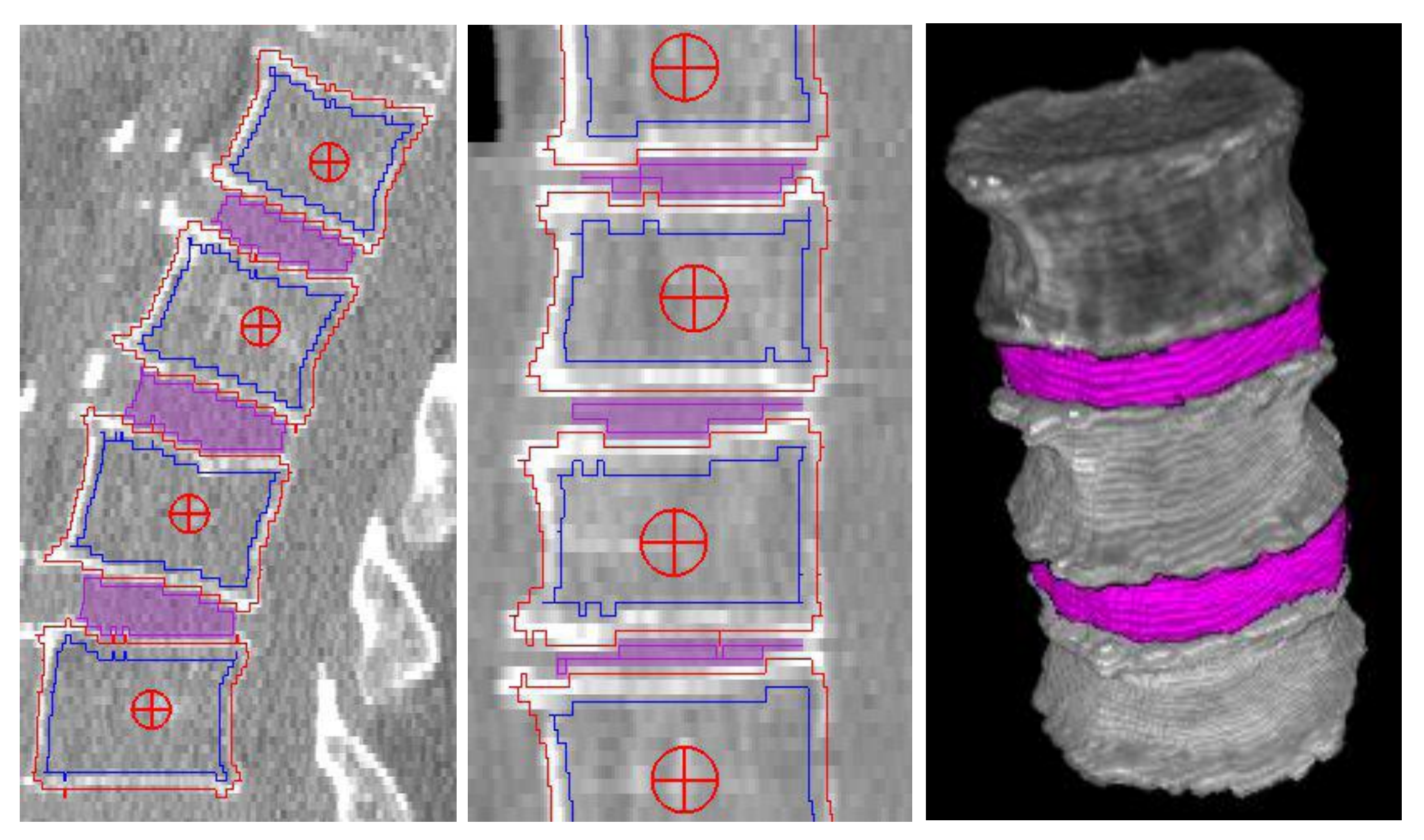

Figure 3: Example of core IDS segmentation on (a): sagittal slice of a The T12-L2.

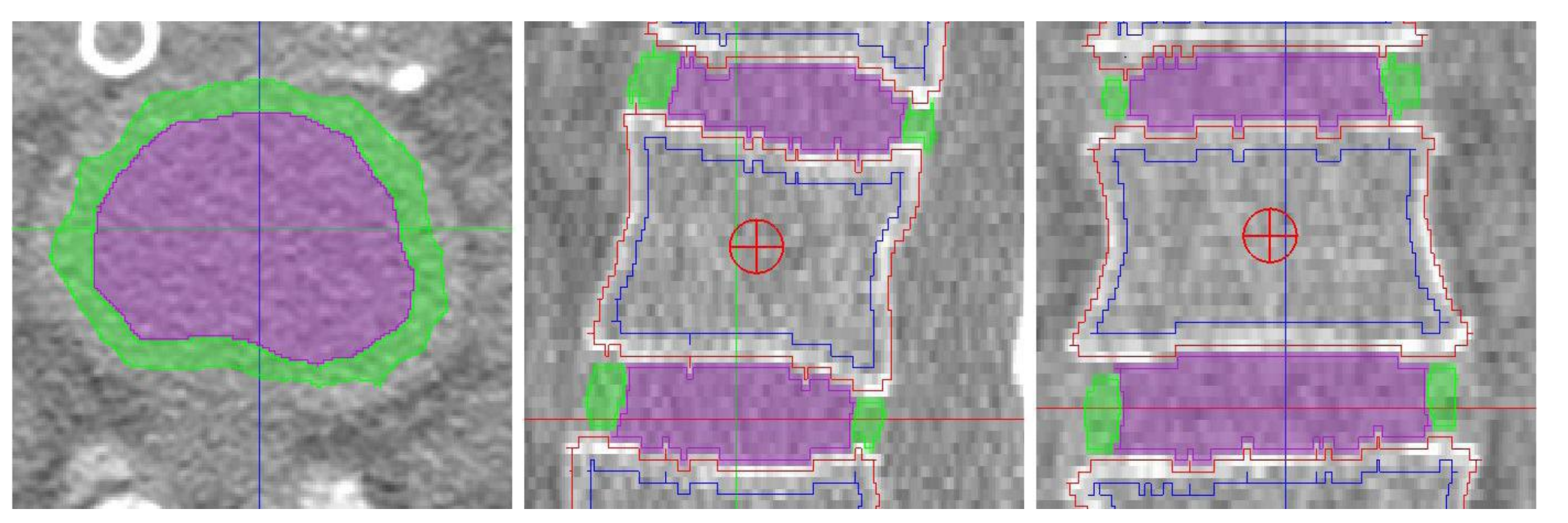

Figure 4: Example of IDS segmentation in three multiplanar reformations: (a) axial, (b) sagittal, and (c) coronal. Core IDS and surrounding extended IDS VOls are shown in purple and green, respectively.

\begin{tabular}{|l|c|c|c|c|c|c|c|c|c|c|}
\hline $\begin{array}{l}\text { \% diff. rel } \\
\text { to T12 }\end{array}$ & T6 & T7 & T8 & T9 & T10 & T11 & L1 & L2 & L3 & L4 \\
\hline N Vert & 6 & 7 & 9 & 11 & 11 & 12 & 10 & 9 & 9 & 3 \\
\hline Int Vol & $-53 \pm 2$ & $-46 \pm 2$ & $-40 \pm 3$ & $-33 \pm 4$ & $-21 \pm 4$ & $-11 \pm 4$ & $+7 \pm 5$ & $+16 \pm 5$ & $+25 \pm 6$ & $+32 \pm 6$ \\
\hline Int BMD & $+8 \pm 6$ & $+9 \pm 7$ & $+6 \pm 5$ & $+9 \pm 7$ & $+10 \pm 8$ & $+7 \pm 6$ & $-3 \pm 5$ & $-5 \pm 10$ & $-5 \pm 14$ & $-17 \pm 26$ \\
\hline Trab BMD & $+15 \pm 22$ & $+13 \pm 14$ & $+6 \pm 12$ & $+6 \pm 13$ & $+9 \pm 9$ & $+8 \pm 5$ & $-9 \pm 9$ & $-14 \pm 11$ & $-17 \pm 15$ & $-30 \pm 24$ \\
\hline IDS Vol & $\mathrm{n} / \mathrm{a}$ & $-69 \pm 13$ & $-58 \pm 8$ & $-49 \pm 14$ & $-41 \pm 7$ & $-18 \pm 21$ & $\mathrm{n} / \mathrm{a}$ & $+94 \pm 54$ & $+126 \pm 72$ & $+108 \pm 21$ \\
\hline
\end{tabular}

Table: Changes (\%) in vertebral integral volume, integral and trabecular BMD, and IDS volume, all normalized to values T12. In the whole population, 12 patients had analyzable T12 resulting in 87 vertebral bodies and 52 IDS in the table.

Integral BMD, $\mathrm{mg} / \mathrm{cm}^{3}$

$\because \because \cdots$

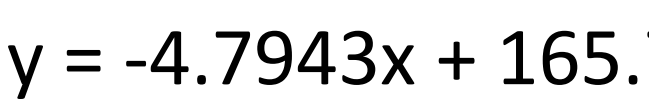

$=0.57$

150

Fig 5: Correlation between vertebral integral BMD and IDS Fig 6: Correlation between vertebral trabecular BMD and IDS volume

Fig 6: Corr
volume

\section{CONCLUSIONS}

A largely automatic segmentation of the thoracic and lumbar spine in CT images including the IDS is feasible. Eventually, this may improve fracture prediction and increase accuracy of finite element models to calculate vertebral strength.

\section{REFERENCES}

1. K. Engelke et al: Reanalysis precision of 3D quantitative computed tomography (QCT) of the spine. Bone 2009, 44

2. Y. M. Baron et al: Intervertebral disc height correlates with vertebral body T-scores in premenopausal and postmenopausal women. Menopause Intl, 2009, 15 